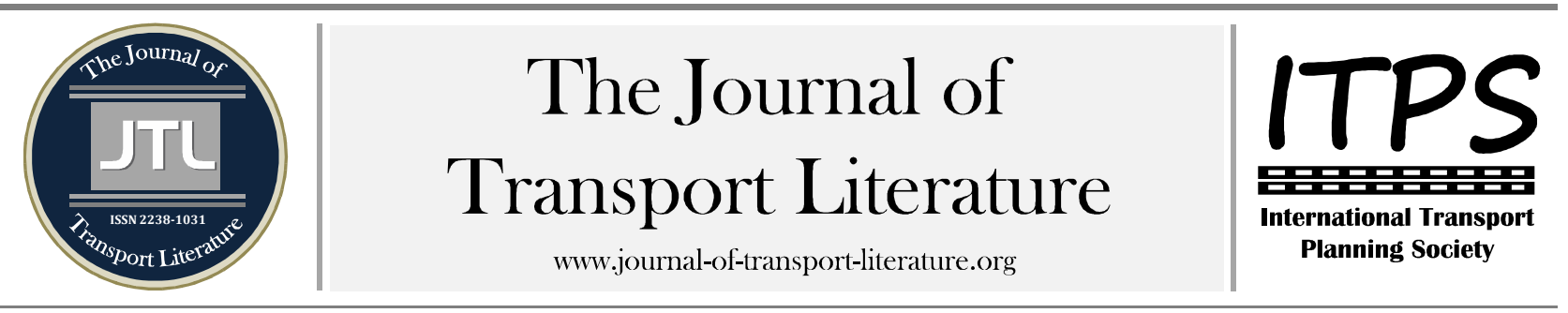

\title{
Modelagem econométrica da demanda em aeroportos privatizados: estudo de caso do Aeroporto Internacional de Confins, Belo Horizonte
}

William Eduardo Bendinelli+; Alessandro V. M. Oliveira

Aeronautics Institute of Technology, São José dos Campos, Brazil

\section{Article Info}

Keywords:

privatização

demanda

transporte aéreo

aeroportos

Submitted 1 May 2014;

received in revised form 4 Jan 2015;

accepted 9 Jan 2015.

Licensed under

Creative Commons

CC-BY $3.0 \mathrm{BR}$.

\section{Resumo}

\begin{abstract}
O objetivo desse artigo é desenvolver um modelo empírico de demanda por viagens de um aeroporto recentemente privatizado pelo governo federal: o Aeroporto Internacional de Confins - Tancredo Neves, em Belo Horizonte. As estimativas da modelagem sugerem que o futuro da concessão do aeroporto está bastante atrelado ao futuro do crescimento econômico do País: com uso de dados setoriais e modelo econométrico com uso de variáveis instrumentais, foi possível estimar elasticidades PIB e preço da demanda em torno de 2 e $-0,15$, respectivamente. A robustez das estimativas obtidas foi checada por meio de um conjunto de análises de sensibilidade com uso de estimadores alternativos. Como conclusão temos que, se por um lado, um baixo crescimento da economia pode afetar negativamente o retorno esperado da concessão dada a alta elasticidade-PIB da demanda aeroportuária, por outro lado, a baixa elasticidadepreço pode gerar pressões dos entes regulados por realinhamentos tarifários que mantenham o equilíbrio econômico-financeiro e a rentabilidade do operador.
\end{abstract}

+ Corresponding author. Praça Marechal Eduardo Gomes, 50. Vila das Acácias, 12228-900. São José dos Campos, SP - Brasil.

E-mail address: wbendinelli@gmail.com.

\section{Introdução}

O presente artigo visa modelar econometricamente a demanda por transporte aéreo no Aeroporto Internacional de Confins - Tancredo Neves, em Belo Horizonte, Minas Gerais. Este aeroporto foi recentemente privatizado, no âmbito do programa de privatizações do governo federal. Como objetivo último, buscaremos estudar os possíveis desdobramentos das relações de demanda aeroportuária com seus principais determinantes - o estado geral da economia (PIB) e a concorrência no setor aéreo (preços das passagens aéreas). 0 modelo empírico obtido permite a extração de estimativas de elasticidadePIB e elasticidade-preço da demanda, que possibilitam análises qualitativas acerca do sucesso do processo de privatização ao longo dos anos, dado que o equilíbrio econômico-financeiro da concessão e a lucratividade da operadora dependerá fundamentalmente da demanda aeroportuária.

Aeroportos têm sido tradicionalmente propriedade e operado pelos governos. No entanto, desde que, no Reino Unido, o governo de Margaret Thatcher vendeu seus principais aeroportos a uma empresa totalmente privada, British Airports Authority (BAA), em um leilão em 1987, a privatização de aeroportos tornou-se gradativamente uma tendência mundial (Oum et al., 2008). Naquele país, todos os aeroportos principais e regionais, com exceção de Manchester, foram privatizados. Na Europa continental, tem ocorrido uma mistura de privatizações parciais, geralmente acompanhada por alguma forma de regulação de preços. A Índia está atualmente no processo de criação de modelos de parcerias públicoprivadas para seus principais aeroportos impondo uma regulamentação de preços. Ao redor do mundo, há vários tipos de propriedade, governança e combinações de regulação de preços, mas aeroportos em diversos países ainda são propriedade pública, incluindo os Estados Unidos e alguns membros da União Europeia.

A questão central do debate na literatura de negócios em aeroportos diz respeito à possibilidade de melhorar ou não a eficiência do aeroporto a partir da privatização, sendo que a capacidade de atrair capital necessário varia de acordo com diferentes tipos de regulação ou combinações de propriedade. Em muitos casos, os governos optaram pela privatização parcial e, nesses casos, eles também impuseram alguma forma de regulação dos preços. No entanto, ainda há governos que estão relutantes em se afastar da propriedade pública ou controle público como, por exemplo, Finlândia, Espanha, Suécia e os EUA. De fato, a importância da aviação no comércio e crescimento econômico significa que os governos buscam selecionar um tipo de governança correta onde uma possível concessão não prejudique o crescimento do setor de aviação, mas, pelo contrário, aumente sua competitividade e gere ganhos de bem-estar.

Diante desse cenário e da necessidade de atrair investimentos, o Brasil adotou, recentemente, uma política de concessões aeroportuárias aos operadores privados. 0 Aeroporto de Confins, em Belo Horizonte, foi um dos aeroportos selecionados para privatização. Segundo a Infraero, os resultados do leilão de privatização, ocorrido em novembro de 2013, apontaram para um ágio moderado de $66 \%$ quando comparado aos outros leilões, mostrando que a demanda por transporte aéreo é volátil às variações de crescimento da economia. Como a demanda é fundamental para o sucesso da privatização, um fraco crescimento econômico acaba afetando negativamente a lucratividade do operador. Entretanto, a inelasticidade da 
demanda aos preços abre espaço para futuros lobbies por aumentos das tarifas aeroportuárias, com vistas a produzir um aumento no retorno do investimento do operador, sobretudo se a economia brasileira não apresentar um ritmo de crescimento significativo.

Assaf e Giffen (2012) examinaram o impacto conjunto que a estrutura de governança e a regulação econômica têm sobre a eficiência do aeroporto. Para os autores, os aeroportos de menor desempenho são aqueles de propriedade do governo e taxa de regulação de retorno. Além disso, concluem que a forma menos eficiente de propriedade é a privatização parcial, independentemente do tipo de regulação. Quanto aos aeroportos congestionados, Lin (2013) mostra que o aeroporto público, que maximiza o bem-estar, define uma taxa abaixo de seus custos operacionais a fim de compensar a elevada taxa cobrada pelos aeroportos privatizados. Se esse déficit fiscal não é permitido, a perda de bem-estar privatizando um hub é menor (maior) do que privatizando aeroportos locais, quando a elasticidade-preço da demanda dos passageiros for pequena (grande), o que é exatamente o mesmo caso quando os aeroportos locais privatizados são substitutos estratégicos (complementos).

Mantin (2012) complementa mostrando que o aeroporto de propriedade de governo não apresenta necessariamente como resultado o ótimo social. Um aeroporto de propriedade do governo, embora procure maximizar o bem-estar, pode apresentar um nível de bem-estar social menor do que se o aeroporto fosse privado. Este é o resultado direto da complementariedade entre aeroportos e os efeitos da gravitação de bem-estar. Por exemplo, se dois aeroportos no sistema são de propriedade de seus governos, cada governo terá o incentivo para privatizar o seu aeroporto, a fim de tirar proveito da gravitação de bem-estar e aumentar o bem-estar local. Se um aeroporto é privado, o governo do país tem o incentivo para privatizar o seu aeroporto, a fim de compensar o efeito negativo da gravitação bem-estar e recuperar algum bem-estar.

Estendendo o conceito de complementariedade de bens para outros setores do aeroporto que apresentam lucro, Czerny (2013) utilizou modelos que capturaram a noção de que a demanda de varejo agregada pode ser independente das atividades de viagem (passageiro a turismo), enquanto a procura por aluguel de automóveis agregada depende das atividades de viagem (passageiro a negócios). Tais resultados podem ser aplicados para outras áreas do aeroporto como, por exemplo, o estacionamento ou a publicidade. A análise mostra que os serviços de varejo podem reduzir os benefícios de regulação de preços no aeroporto, enquanto eles também podem levar ao congestionamento excessivo do aeroporto. Aluguel de carros, por outro lado, pode aumentar os benefícios da regulação, porque eles podem aumentar ainda mais a tarifa aeronáutica já excessiva e a cobrança de aluguel de carro particular pode ser excessivo também.

Dessa forma, o presente trabalho está assim dividido: na Seção 1 será apresentado as bases de dados utilizadas no artigo bem como análise descritiva dos dados. A Seção 2 o modelo econométrico e os resultados das estimações da demanda aeroportuária para a cidade de Belo Horizonte. Por fim, são apresentadas as conclusões do artigo.

\section{Apresentação da base de dados utilizadas na estimação da função demanda}

A base de dados utilizada no estudo constitui-se de dados secundários mensais de janeiro de 2000 a junho de 2012 . As principais fontes de dados de demanda correspondem ao movimento de passageiros da Empresa Brasileira de Infraestrutura Aeroportuária (Infraero) e do conjunto de Relatórios de Tarifas Aéreas Domésticas da Agência Nacional de Aviação Civil (ANAC) em valores nominais. Como indicador de renda, utilizou-se o Produto Interno Bruto mensal, disponibilizado pelo Banco Central do Brasil com valores nominais. Como dados de taxa de inflação, utilizou-se o Índice Nacional de Preços ao Consumidor Amplo (IPCA), obtido no Instituto Brasileiro de Geografia e Estatística (IBGE).

Em termos macroeconômicos, o período analisado apresenta características importantes que impactaram o setor aéreo. Principalmente ao longo do Governo Lula (2003-2010), a Formação Bruta de Capital Físico intensificou-se, ampliando o Produto Interno Bruto do País. Nesse mesmo período, com a política de transferência de renda associada a maior facilidade de acesso ao crédito, o setor aéreo, através da popularização das passagens aéreas, pôde absorver a crescente demanda para esse tipo de transporte que, em muitos casos, tornou-se um bem substituto ao transporte rodoviário. Por outro lado, como fatores impactantes de custo para as empresas aéreas, o preço do barril de petróleo chegou ao valor mais alto de sua série na crise financeira de 2008 após ameaças do Irã bloquear o trânsito de petróleo no Golfo Pérsico, o temor de uma nova guerra no Oriente Médio e as perturbações da Nigéria. Além do preço do barril de petróleo, a taxa de câmbio tem-se apreciado desde o início do Governo Lula.

A Tabela 1 apresenta o resumo das principais variáveis utilizadas para estimar o modelo de demanda.

\begin{tabular}{llllll}
\multicolumn{7}{c}{ Tabela 1 - Estatísticas das variáveis analisadas. } \\
\hline Variável & Unidade & Média & Desv.Pad. & Mínimo & Máximo \\
\hline Passageiros domésticos & Pax & 418.981 & 196.925 & 193.689 & 972.685 \\
PIB & R $\$$ & 2.867 .312 & 561.648 & 2.005 .540 & 3.946 .980 \\
Receita média por RPK & R\$ & 66,37 & 21,9859 & 30,04 & 113,56 \\
Petróleo WTI & US\$ por galão & 58,74 & 28,0674 & 19,39 & 133,88 \\
Taxa de câmbio efetivo & US\$ & 1,4838 & 0,7997 & 0,5054 & 3,3166 \\
\hline
\end{tabular}

Nota-se na Tabela 1 que durante o período analisado, as variáveis apresentaram grande oscilação em torno da média. 0 número de passageiros embarcados e desembarcados domésticos totais (paxdom) variou de um mínimo de 193,7 mil passageiros e atingiu um máximo de 972,7 mil passageiros domésticos totais, num crescimento que se intensificou após o ano de 2009 e congestionou os aeroportos analisados. Nesse mesmo sentido, a receita média doméstica por passageiroquilômetro transportado pago (yielddom), que atingiu o valor máximo de $\mathrm{R} \$ 113,56$ centavos em dezembro de 2002 , declinou-se substancialmente após a guerra de preços que marcou o setor nesse período, e atingiu seu valor mínimo em julho de 2011 de $R \$ 30,05$. Assim, percebe-se uma tendência negativa desde a entrada da Gol no mercado e o início da guerra de preços, acirrando-se com a entrada da Azul a partir de 2009. Com o fim do codeshare Varig-TAM o preço das passagens aéreas reduziram-se bastante. Além disso, ressalta-se que o patamar do yielddom em 2008 está mais associado a fatores como depreciação da taxa de câmbio, relaxamentos regulatórios e incentivos governamentais em resposta à crise financeira do que propriamente a um efeito ligado à concorrência do setor. 


\section{Estimativa e análise dos resultados da demanda aeroportuária para Belo Horizonte}

Para estimar os parâmetros da regressão de demanda aeroportuária, utilizou-se o GMM (Método dos Momentos Generalizados, ou "Generalized Method of Moments") em dois estágios dado pela Equação (1), que precisa ser corrigido por apresentar problemas de heteroscedasticidade e autocorrelação das variáveis, além de existir endogeneidade da variável explicativa yielddom. Dessa forma, como estratégia de identificação da demanda, assume-se que os fatores não-observáveis de demanda e preço, extraídos por meio dos valores verdadeiros dos parâmetros, são média-independentes dos vetores de características dos produtos e de custos. Assim, a Equação (2) mostra como instrumentos de demanda, o conjunto de deslocadores de custos incluídos nessa equação e excluídos da demanda.

$$
\begin{gathered}
\ln (\text { paxdom })=\beta_{1} \text { gdp }+\beta_{2} \text { yielddom }{ }^{*}+\beta_{3} y 0203+\beta_{4} y 0911+\sum_{i=1}^{12} \beta_{i} m_{i}+u \\
\text { yielddom }^{*}=\delta_{1} \text { yielddom }_{t-1}+\delta_{2} \text { poilw }+\delta_{3} \text { poil }_{t-1}+\delta_{4} \text { indusdeff }+\varepsilon
\end{gathered}
$$

onde:

- In(paxdom): logaritmo neperiano do número de passageiros embarcados e desembarcados domésticos totais obtidos da Infraero.

- $g d p$ : Produto Interno Bruto. Valores em bilhões de reais deflacionados a valor presente obtidos do Bacen.

- yielddom: receita média doméstica por passageiro-quilômetro transportado pago. Valores em reais deflacionados a valor presente obtidos da ANAC.

- y0203: dummy deslocadora de intercepto, sendo a soma dos anos de 2002 e 2003 que representa o efeito do codeshare Varig-TAM.

- y0911: dummy deslocadora de intercepto, sendo a soma dos anos de 2009 e 2011 que representa o crescimento acelerado do número de passageiros embarcados e desembarcados domésticos totais.

- $m_{i}$ : dummy de sazonalidade que apresenta como referência o mês de janeiro;

- poilw: preço do barril de petróleo WTI em dólares obtidos do Ipea.

- indusdeff: índice de taxa de câmbio real efetiva, em valores constantes obtidos do Ipea.

A Tabela 2 apresenta o resultado das estimações do modelo econométrico apresentado pela Equação (1) para a demanda aeroportuária do Aeroporto Internacional de Confins - Tancredo Neves. Nela, são apresentadas tanto as estimativas dos

\begin{tabular}{|c|c|c|c|c|c|c|}
\hline \multirow[b]{2}{*}{ yielddom } & \multicolumn{2}{|c|}{ Coeficiente } & \multirow{2}{*}{$\begin{array}{c}\text { Erros-padrões } \\
0,0010\end{array}$} & \multirow{2}{*}{$\begin{array}{c}\text { Elasticidade } \\
-0,1304\end{array}$} & \multirow{2}{*}{\multicolumn{2}{|c|}{$\begin{array}{c}\text { Erros-padrões } \\
0,065\end{array}$}} \\
\hline & $-0,002$ & *** & & & & \\
\hline gdp & 0,007 & $* * *$ & 0,0005 & 2,0544 & $* * *$ & 0,144 \\
\hline y0203 & 0,094 & $* * *$ & 0,0333 & 0,0152 & $* * *$ & 0,005 \\
\hline y0911 & $-0,004$ & & 0,0675 & $-0,0006$ & & 0,011 \\
\hline m_2 & $-0,063$ & $* *$ & 0,0253 & $-0,0055$ & $* *$ & 0,002 \\
\hline m_6 & $-0,114$ & $* *$ & 0,0477 & $-0,0099$ & $* *$ & 0,004 \\
\hline m_11 & $-0,174$ & $* * *$ & 0,0382 & $-0,0140$ & $* * *$ & 0,003 \\
\hline m_12 & $-0,127$ & $* * *$ & 0,0368 & $-0,0102$ & $* * *$ & 0,003 \\
\hline $\mathbf{R}$ quadrado & 0,9276 & & & & & \\
\hline F - Estatística & 45,2915 & & & & & \\
\hline F - P-valor & 0,0000 & & & & & \\
\hline KP - Estatística & 14,1047 & & & & & \\
\hline KP - P-valor & 0,0070 & & & & & \\
\hline J - Estatística & 3,0373 & & & & & \\
\hline J - P-valor & 0,3859 & & & & & \\
\hline
\end{tabular}
parâmetros como as suas elasticidades.

Notas: (i) Estimativas de elasticidades apresentadas na média amostral. (ii)Erros padrões estimados em colchetes.

(iii) Representações de $\mathrm{p}$-valor: ${ }^{* * *} \mathrm{p}<0,01$; $^{* *} \mathrm{p}<0,05{ }^{*} \mathrm{p}<0,10$.

A análise da Tabela 2 mostra, primeiramente, que o valor de R quadrado (R-sq) alto representa boa qualidade do ajuste da regressão e a estatística F aprova a significância conjunta dos regressores. Como se pode observar, aceita-se a hipótese nula de que não existem não-linearidades negligenciadas ao nível de significância de 0,05 . Seguindo a análise, a estatística rk de Kleibergen-Paap (KP) rejeita a hipótese nula de que a equação é subespecificada, isto é, a matriz possui posto completo e identificação. Por fim, para o teste J de Hansen aceita-se a hipótese nula de que os instrumentos utilizados são instrumentos válidos, isto é, ortogonais ao vetor de resíduos. Portanto, o modelo utilizado apresenta robustez após a correção da heteroscedasticidade e autocorrelação.

Em relação aos coeficientes e elasticidades estimadas, em geral, a maioria das variáveis de interesse apresentou coeficientes estimados que são, ao mesmo tempo, estatisticamente significantes e com o sinal consistente com o esperado. A elasticidade-preço da demanda $(-0,1304)$, mostra uma demanda inelástica ao preço, uma vez que o mercado de transporte aéreo é marcado fortemente pelo segmento corporativo, insensível a preços. Adicionalmente, deve ser levado em conta que viagens de negócio são planejadas com pouca antecedência e financiadas pelas empresas, que em geral privilegiam a variável tempo. Além disso, como se pode observar, o Aeroporto de Confins apresenta como principais rotas de destinos as pontes aéreas que apresentam substancialmente menor sensibilidade ao preço. Assim, uma elevação de $1 \%$ no preço das passagens aéreas reduz a demanda em $0,13 \%$. Por outro lado, a elasticidade-renda da demanda apresentou-se bastante sensível a alterações a renda e que pode estar relacionado à popularização das passagens aéreas que absorveu os novos consumidores da classe C. Ou seja, uma elevação de 1\% da renda média dos passageiros eleva a demanda por transporte aéreo em 2,05\%. Nesse sentido, pode-se verificar que a demanda no Aeroporto de Confins está bastante relacionada com o crescimento econômico. Nesse sentido, se houver baixo crescimento econômico, a demanda por viagens e o próprio retorno esperado do investimento poderão ser bastante impactados. 


\subsection{Análise de sensibilidade das estimativas com uso de estimadores diferentes}

A robustez das estimativas obtidas do modelo de demanda aeroportuária foi checada por meio de um conjunto de análises de sensibilidade com uso de estimadores alternativos, conforme descrevemos a seguir. Adicionalmente à especificação do modelo através do método GMM, utilizou-se outros métodos e estimadores para efeito de comparação entre os modelos. A Tabela 3 apresenta os resultados das estimações das elasticidades da modelagem empírica da demanda para os modelos 2SLS, GMM2S, LIML e CUE. Segundo as recomendações de Angrist e Pischke (2008), se as comparações entre os métodos 2SLS com o estimador LIML (Limited Information Maximum Likelihood) resultarem parecidos, pode-se aceitar os instrumentos e os resultados da estimação dos parâmetros da regressão obtidos com 2SLS. Segundo os autores, esta consideração deve-se ao fato de LIML produzir resultados com menor viés do que 2SLS representando parâmetros confiáveis e constituindo-se em uma boa referência de adequação do método de regressão utilizado.

Tabela 3. Resultados das estimações de demanda: especificações alternativas.

\begin{tabular}{|c|c|c|c|c|c|c|c|c|}
\hline \multirow{2}{*}{ Variável } & \multicolumn{8}{|c|}{ Modelo } \\
\hline & \multicolumn{2}{|l|}{ 2SLS } & \multicolumn{2}{|l|}{ GMM2S } & \multicolumn{2}{|l|}{ LIML } & \multicolumn{2}{|l|}{ CUE } \\
\hline \multirow[t]{2}{*}{ yielddom } & $-0,1070$ & & $-0,1304$ & $* *$ & $-0,1084$ & & $-0,1381$ & $* *$ \\
\hline & {$[0,088]$} & & {$[0,065]$} & & {$[0,089]$} & & {$[0,066]$} & \\
\hline \multirow[t]{3}{*}{ gdp } & 1,9981 & $* * *$ & 2,0544 & $* * *$ & 1,9974 & $* * *$ & 2,1614 & $* * *$ \\
\hline & {$[0,158]$} & & {$[0,144]$} & & {$[0,158]$} & & {$[0,157]$} & \\
\hline & {$[0,003]$} & & {$[0,003]$} & & {$[0,003]$} & & {$[0,003]$} & \\
\hline \multirow[t]{2}{*}{ y0203 } & 0,0118 & & 0,0152 & $* * *$ & 0,0118 & & 0,0153 & $* * *$ \\
\hline & {$[0,008]$} & & {$[0,005]$} & & {$[0,008]$} & & {$[0,006]$} & \\
\hline \multirow[t]{2}{*}{ y0911 } & 0,0016 & & $-0,0006$ & & 0,0015 & & $-0,0081$ & $* * *$ \\
\hline & {$[0,011]$} & & {$[0,011]$} & & {$[0,011]$} & & {$[0,011]$} & \\
\hline $\mathbf{R}$ quadrado & 0,9291 & & 0,9276 & & 0,9291 & & 0,9218 & \\
\hline F - Estatística & 0,1157 & & 0,1169 & & 0,1157 & & 0,1215 & \\
\hline F - P-valor & 39,2220 & & 45,2915 & & 39,2838 & & 59,6224 & \\
\hline KP - Estatística & 0,0000 & & 0,0000 & & 0,0000 & & 0,0000 & \\
\hline KP - P-valor & 14,1047 & & 14,1047 & & 14,1047 & & 14,1047 & \\
\hline J - Estatística & 0,0070 & & 0,0070 & & 0,0070 & & 0,0070 & \\
\hline J - P-valor & 3,0373 & & 3,0373 & & 3,0374 & & 2,3288 & \\
\hline R quadrado & 0,3859 & & 0,3859 & & 0,3859 & & 0,5070 & \\
\hline
\end{tabular}

Notas: (i) Estimativas de elasticidades apresentadas na média amostral. (ii)Erros padrões estimados em colchetes.

(iii) Representações de $\mathrm{p}$-valor: ${ }^{* * *} \mathrm{p}<0,01 ;{ }^{* *} \mathrm{p}<0,05 ;{ }^{*} \mathrm{p}<0,10$. (iv) Dummies de sazonalidade omitidas.

A análise da Tabela 3 permite verificarmos o viés de simultaneidade quando comparamos os modelos. Embora as especificações de CUE e LIML não fornecem mais ganhos de eficiência assintótica do que o GMM2S, seu desempenho para amostras finitas pode ser superior. Nesse sentido, há evidências sugerindo que CUE e LIML apresentam melhores desempenhos do que o GMM2S na presença de instrumentos fracos. A desvantagem do CUE, em geral, está no fato de requerer alguma forma de otimização numérica, enquanto que para o LIML a otimização não é necessária. No entanto, o último acaba muitas vezes apresentando uma hipótese forte para distúrbios i.i.d. Portanto, pode não haver robustez no modelo por causa dos instrumentos serem fracos. Ressalta-se, por exemplo, que a variável independente yielddom apresentou coeficiente negativo, como o esperado, embora seja somente significativa nos modelos GMM2S e CUE. Por se tratar de uma variável-chave na estimação da demanda, não apresentar significância estatística torna-se um problema. Por outro lado, a variável gdp é significante para todos os modelos. Pode-se notar também que o modelo 2SLS é o modelo em que as elasticidades próprias são as menores, o contrário do que ocorre com o modelo CUE. Dessa forma, o modelo proposto, GMM2S, apresentou estimativas de elasticidades e de variabilidade que ficaram próximas aos valores extremos obtidos com os demais modelos, o que pode ser um indicativo de viés.

\section{Conclusão}

O objetivo desse artigo foi estimar a demanda aeroportuária para o Aeroporto de Confins e melhor entender a relação do aeroporto com sua demanda no ambiente pós-privatização. Para isso, desenvolveu-se uma modelagem econométrica de demanda aeroportuária e estimou-se as elasticidades preço e renda da demanda como forma de analisar tanto a alocação como a atração de investimentos para o setor aéreo.

0 modelo econométrico apontou uma demanda inelástica ao preço, uma vez que o mercado de transporte aéreo é ainda marcado pelo segmento corporativo, em geral bastante insensível aos preços. Assim, uma elevação de $1 \%$ no preço das passagens aéreas reduz a demanda em 0,13\%. Por outro lado, a elasticidade-renda da demanda apresentou-se razoavelmente sensível a alterações a renda e que está relacionado à popularização das passagens aéreas que absorveu os novos consumidores da classe C. Ou seja, uma elevação de $1 \%$ da renda média dos passageiros eleva a demanda por transporte aéreo em 2,05\%. Nesse sentido, temos que o sucesso da privatização está consideravelmente relacionado ao crescimento econômico do País. Se por um lado, o baixo crescimento da economia tem afetado negativamente a possibilidade de lucratividade, por outro lado, a inelasticidade da demanda aos preços abre espaço para lobbies dos entes privados regulados que obtiveram concessão de aeroportos, em busca de realinhamentos tarifários. A regulação aeroportuária deverá cuidar de acomodar essas pressões de forma a manter o foco não apenas no bem-estar do consumidor, mas na garantia dos investimentos setoriais nessa importante infraestrutura básica do País. 


\section{Referências}

Angrist, J. D., \& Pischke, J. S. (2008). Mostly Harmless Econometrics. United Kingdom: Princeton University Press.

Assaf, A.G., \& Gillen, D. (2012). Measuring the joint impact of governance form and economic regulation on airport efficiency. European Journal of Operation Research, 220(1), 187-198.

Czerny, A.I. (2013). Public versus private airport behavior when concession revenues exist. Economics of Transportation, 2(1), 38-46.

Lin, M. H. (2013). Airport privatization in congested hub-spoke networks. Transportation Research Part B, 54, 51-67.

Mantin, B. (2012). Airport complementarity: Private vs. government ownership and welfare gravitation. Transportation Research Part B, 46(3), 381-388.

Oum, T. H., Yan, J., \& Yu, C. (2008). Ownership forms matter for airport efficiency: a stochastic frontier investigation of worldwide airports. Journal of Urban Economics, 64(2), 422-435.

\section{Abstract}

The purpose of this paper is to develop an empirical model of demand for travel to the airport recently privatized by the federal government: the Confins-Tancredo Neves International Airport, Belo Horizonte, Brazil. Estimates of the modelling suggest that the future of the airport concession is considerably related to the economic growth of the country: with the use of industry data and econometric model of instrumental variables, it was possible to estimate GDP and price elasticities of demand around 2 and -0.15 , respectively. The robustness of the estimates was checked by means of a set of sensitivity analyses using alternative estimators. In conclusion, we have, on one hand, a low growth economy may negatively affect the expected return of the concession given to high GDP elasticity of demand of airport, on the other hand, the low price elasticity may generate pressures from the regulated entities for tariff increases that maintain economic and financial stability and profitability of the operator.

Key words: privatization, demand, air transport, airports. 